PROCEEDINGS OF THE

AMERICAN MATHEMATICAL SOCIETY

Volume 138, Number 6, June 2010, Pages 1897-1906

S 0002-9939(10)10309-8

Article electronically published on February 5, 2010

\title{
A COMBINATORIAL CONSTRUCTION OF HIGH ORDER ALGORITHMS FOR FINDING POLYNOMIAL ROOTS OF KNOWN MULTIPLICITY
}

\author{
YI JIN AND BAHMAN KALANTARI \\ (Communicated by Peter A. Clarkson)
}

\begin{abstract}
We construct a family of high order iteration functions for finding polynomial roots of a known multiplicity $s$. This family is a generalization of a fundamental family of high order algorithms for simple roots that dates back to Schröder's 1870 paper. It starts with the well known variant of Newton's method $\hat{B}_{2}(x)=x-s \cdot p(x) / p^{\prime}(x)$ and the multiple root counterpart of Halley's method derived by Hansen and Patrick. Our approach demonstrates the relevance and power of algebraic combinatorial techniques in studying rational root-finding iteration functions.
\end{abstract}

\section{INTRODUCTION}

In 4 we introduced symmetric functions to the study of iterative root-finding algorithms, thus revealing the combinatorial nature of rational root-finding iteration functions. In particular, we gave a combinatorial interpretation of the Basic Family - a fundamental family of high order algorithms for finding simple roots of a polynomial. The Basic Family first appeared in Schröder's 1870 paper [14] and was later rediscovered many times under different representations. In one representation, it is known as König's family (see [1]). In [7, Kalantari et al. gave a purely algebraic derivation of the Basic Family and revealed many interesting minimality and uniqueness properties of this family. For some related works in this direction, see [8, [9, 10, 11] and [6].

In this article we demonstrate the power of our combinatorial approach by deriving an efficient algorithm to construct a generalized Basic Family for roots with a known multiplicity. Our exposition is organized as follows: In Section 2 we give a brief review of relevant results from [4. In Section 3 we present a combinatorial construction of the generalized Basic Family and prove its convergence property. In Section 4 we show the connection between the Basic Family and its generalization.

Received by the editors December 4, 2007.

2010 Mathematics Subject Classification. Primary 05E05, 05A15, 65D15; Secondary 65Q05, $65 \mathrm{H} 05$

Key words and phrases. Symmetric functions, generating functions, recurrence relation, multiple roots, root-finding, iteration functions.

The main idea of this article was developed in the first author's Ph.D. dissertation [5], supervised by the second author. 


\section{Preliminaries}

First we recall some notation and results from the theory of symmetric functions. For a definitive treatment on this subject, see MacDonald [13].

For each integer $r \geq 1$, the $r$-th elementary symmetric function $e_{r}$ is the sum of all products of $r$ distinct variables $x_{i}$, the $r$-th complete symmetric function $h_{r}$ is the sum of all monomials of total degree (the sum of exponents of all its variables) $r$ in the variables $x_{1}, x_{2}, \ldots$, and the $r$-th power sum is the sum of $r$-th power of $x_{1}, x_{2}, \ldots$ :

$$
\begin{aligned}
e_{r} & =\sum_{i_{1}<i_{2}<\cdots<i_{r}} x_{i_{1}} x_{i_{2}} \ldots x_{i_{r}}, \\
h_{r} & =\sum_{a_{1}+a_{2}+\cdots=r} x_{1}^{a_{1}} x_{2}^{a_{2}} \ldots, \\
q_{r} & =\sum_{i \geq 1} x_{i}^{r} .
\end{aligned}
$$

Additionally, define $e_{0}=h_{0}=1$.

The generating functions for $e_{r}, h_{r}$ and $q_{r}$ are

$$
\begin{aligned}
E(t) & =\sum_{r \geq 0} e_{r} t^{r}=\prod_{i \geq 1}\left(1+x_{i} t\right), \\
H(t) & =\sum_{r \geq 0} h_{r} t^{r}=\prod_{i \geq 1}\left(1-x_{i} t\right)^{-1}, \\
Q(t) & =\sum_{r \geq 1} q_{r} t^{r-1}=\sum_{i \geq 1} \frac{x_{i}}{1-x_{i} t} .
\end{aligned}
$$

Thus, $H(t)=E(-t)^{-1}$ and

$$
Q(t)=\frac{d}{d t} \log H(t)=\frac{H^{\prime}(t)}{H(t)}=\frac{E^{\prime}(-t)}{E(-t)},
$$

where $E^{\prime}(-t)$ denotes the derivative of $E$ evaluated at $-t$.

The above relations among generating functions imply the following recursions:

$$
\begin{array}{r}
\sum_{r=0}^{n}(-1)^{r} e_{r} h_{n-r}=0 \quad \text { for } \quad n=1,2, \ldots, \\
n h_{n}=\sum_{r=1}^{n} q_{r} h_{n-r} \quad \text { for } \quad n=1,2, \ldots, \\
n e_{n}=\sum_{r=1}^{n}(-1)^{r-1} q_{r} e_{n-r} \quad \text { for } \quad n=1,2, \ldots
\end{array}
$$

We now make a connection between symmetric functions and root-finding algorithms.

Let $p(x)$ be a polynomial of degree $n$ with complex coefficients and let $\theta_{1}, \ldots, \theta_{n}$ be its complex roots. Then,

$$
p(x)=c \prod_{i=1}^{n}\left(x-\theta_{i}\right),
$$

where $c$ is the leading coefficient of $p(x)$.

Define $r_{j}=1 /\left(x-\theta_{j}\right), j=1, \ldots, n$. Then we have 
Proposition 2.1 (Jin and Kalantari 4, Lemma 3.1).

$$
p^{(i)}(x)=i ! p(x) e_{i}\left(r_{1}, \ldots, r_{n}\right),
$$

where $e_{i}$ is the $i$-th elementary symmetric function.

For each integer $m \geq 2$, define the $m$-th order Basic Family iteration function

$$
B_{m}(x)=x-\frac{h_{m-2}\left(r_{1}, \ldots, r_{n}\right)}{h_{m-1}\left(r_{1}, \ldots, r_{n}\right)} .
$$

This is equivalent to the original definition of Basic Family given by Kalantari et al. 7.

Proposition 2.2 (Jin and Kalantari 4, Corollary 3.4). $B_{m}(x)$ has an $m$-th order of convergence to a simple root and linear convergence to a multiple root.

Proposition 2.1 and the recurrence relation between $h_{r}$ and $e_{r}$ give rise to an efficient algorithm to compute $B_{m}$.

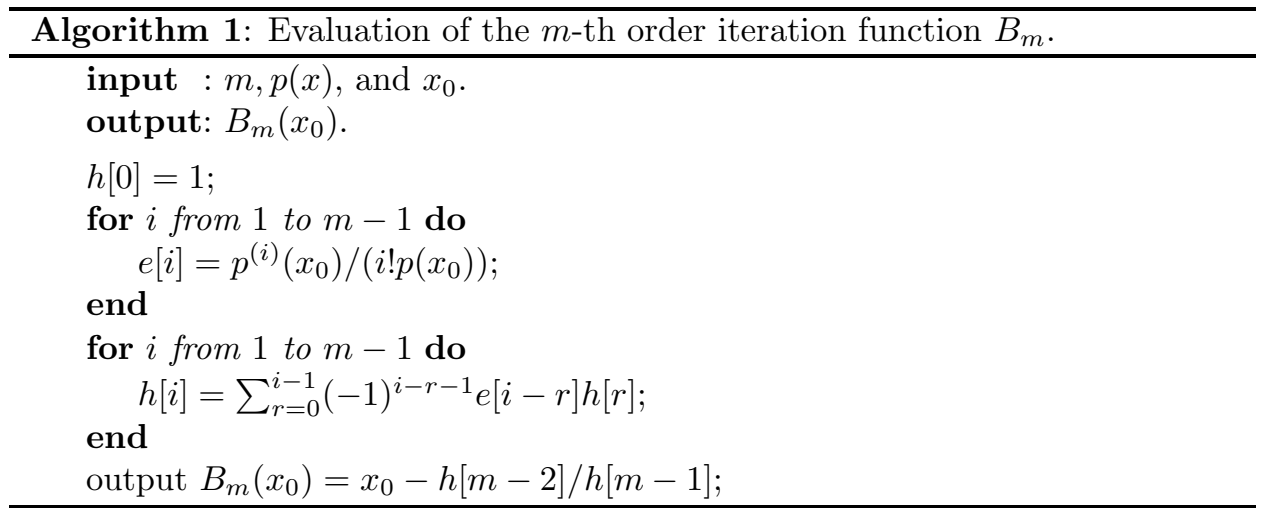

The first three members of the Basic Family are

$$
B_{2}(x)=x-\frac{p(x)}{p^{\prime}(x)}
$$

which is the well known Newton's method,

$$
B_{3}(x)=x-\frac{2 p(x) p^{\prime}(x)}{2 p^{\prime}(x)^{2}-p(x) p^{\prime \prime}(x)},
$$

which is Halley's method, and the 4th order method

$$
B_{4}(x)=x-\frac{6 p(x) p^{\prime}(x)^{2}-3 p(x)^{2} p^{\prime \prime}(x)}{p(x)^{2} p^{\prime \prime \prime}(x)+6 p^{\prime}(x)^{3}-6 p(x) p^{\prime}(x) p^{\prime \prime}(x)} .
$$

For a root of multiplicity $s \geq 2$, there is a well known generalization of the 2nd order Newton's method:

$$
\hat{B}_{2}(x)=x-s \frac{p(x)}{p^{\prime}(x)} .
$$

A generalization of the 3rd order Halley's method was derived by Hansen and Patrick [3, Equation (8.2)].

In this article, we shall show that such generalization exists for all Basic Family iteration functions, and it can be computed efficiently due to its simple combinatorial characterization. 


\section{3. $\left\{\hat{B}_{m}\right\}_{m=2}^{\infty}$ - A FAMILY OF HIGH ORDER METHODS \\ FOR ROOTS OF MULTIPLICITY $s$}

The key ingredient in our combinatorial construction is a generalization of power sums and complete symmetric functions.

Define the generalized power sums

$$
\hat{q}_{r}=\frac{q_{r}}{s}=\frac{1}{s} \sum_{i \geq 1} x_{i}^{r} \quad \text { for } \quad r=1,2, \ldots
$$

The generalized complete symmetric functions $\hat{h}_{r}$ are defined through the following recursion:

$$
\begin{aligned}
& \hat{h}_{0}=1, \\
& \hat{h}_{r}=\frac{1}{r} \sum_{i=0}^{r-1} \hat{q}_{r-i} \hat{h}_{i} \quad \text { for } \quad r=1,2, \ldots
\end{aligned}
$$

Define the $m$-th order generalized Basic Family iteration functions

$$
\hat{B}_{m}(x)=x-\frac{\hat{h}_{m-2}\left(r_{1}, \ldots, r_{n}\right)}{\hat{h}_{m-1}\left(r_{1}, \ldots, r_{n}\right)} \quad \text { for } \quad m=2,3, \ldots
$$

Note that when $s=1, \hat{B}_{m}$ reduces to $B_{m}$, an $m$-th order method for simple roots.

In the following subsection, we shall show that $\hat{B}_{m}$ is an $m$-th order method for roots of multiplicity $s$.

3.1. Proof of convergence property of $\hat{B}_{m}$. Recall that the falling factorial is defined as

$$
(x)_{0}=1 \quad \text { and } \quad(x)_{n}=x(x-1) \ldots(x-n+1) \text { for } n=1,2, \ldots .
$$

We first express $\hat{h}_{k}\left(r_{1}, \ldots, r_{n}\right)$ as a polynomial in $r_{1}$.

Lemma 3.1. Assume that $\theta_{1}$ is a root of multiplicity $s^{\prime}$, i.e., $\theta_{1}=\theta_{2}=\cdots=\theta_{s^{\prime}}$, and $\theta_{i} \neq \theta_{1}$ for $i=s^{\prime}+1, \ldots, n$. Define $r_{j}=1 /\left(x-\theta_{j}\right), j=1, \ldots, n$. Then for every integer $k \geq 0$,

$$
\hat{h}_{k}\left(r_{1}, \ldots, r_{n}\right)=\sum_{i=0}^{k} \frac{\left(k-i+s^{\prime} / s-1\right)_{k-i}}{(k-i) !} \hat{h}_{i}\left(r_{s^{\prime}+1}, \ldots, r_{n}\right) r_{1}^{k-i} .
$$

Proof. By induction on $k$.

For $k=0$, (3.4) holds trivially. Now assume that (3.4) holds for $k<m, m \in \mathbb{Z}^{+}$. Then for $k=m$, by (3.2) we have the following recursion:

$$
\hat{h}_{m}\left(r_{1}, \ldots, r_{n}\right)=\frac{1}{m} \sum_{j=0}^{m-1} \hat{q}_{m-j}\left(r_{1}, \ldots, r_{n}\right) \hat{h}_{j}\left(r_{1}, \ldots, r_{n}\right) .
$$

By (3.1) and induction assumption, we have

$$
\begin{array}{r}
\hat{q}_{m-j}\left(r_{1}, \ldots, r_{n}\right)=\frac{s^{\prime}}{s} r_{1}^{m-j}+\hat{q}_{m-j}\left(r_{s^{\prime}+1}, \ldots, r_{n}\right) \quad \text { for } m-j>0, \\
\hat{h}_{j}\left(r_{1}, \ldots, r_{n}\right)=\sum_{i=0}^{j} \frac{\left(j-i+s^{\prime} / s-1\right)_{j-i}}{(j-i) !} \hat{h}_{i}\left(r_{s^{\prime}+1}, \ldots, r_{n}\right) r_{1}^{j-i} \text { for } j<m .
\end{array}
$$


Plugging the above identities into (3.5), multiplying out, and collecting like terms of powers of $r_{1}$, we get

$$
\hat{h}_{m}\left(r_{1}, \ldots, r_{n}\right)=\frac{1}{m} \sum_{i=0}^{m} c_{i} r_{1}^{m-i}
$$

where

$$
\begin{aligned}
c_{i} & =\frac{s^{\prime}}{s} \hat{h}_{i}\left(r_{s^{\prime}+1}, \ldots, r_{n}\right) \sum_{j=i}^{m-1} \frac{\left(j-i+s^{\prime} / s-1\right)_{j-i}}{(j-i) !} \\
& +\frac{\left(m-i+s^{\prime} / s-1\right)_{m-i}}{(m-i) !} \sum_{j=1}^{i} \hat{h}_{i-j}\left(r_{s^{\prime}+1}, \ldots, r_{n}\right) \hat{q}_{j}\left(r_{s^{\prime}+1}, \ldots, r_{n}\right) \\
& =\frac{\left(m-i+s^{\prime} / s-1\right)_{m-i}}{(m-i-1) !} \hat{h}_{i}\left(r_{s^{\prime}+1}, \ldots, r_{n}\right) \\
& +\frac{\left(m-i+s^{\prime} / s-1\right)_{m-i}}{(m-i) !} i \hat{h}_{i}\left(r_{s^{\prime}+1}, \ldots, r_{n}\right) \\
& =m \frac{\left(m-i+s^{\prime} / s-1\right)_{m-i}}{(m-i) !} \hat{h}_{i}\left(r_{s^{\prime}+1}, \ldots, r_{n}\right) .
\end{aligned}
$$

Thus,

$$
\hat{h}_{m}\left(r_{1}, \ldots, r_{n}\right)=\sum_{i=0}^{m} \frac{\left(m-i+s^{\prime} / s-1\right)_{m-i}}{(m-i) !} \hat{h}_{i}\left(r_{s^{\prime}+1}, \ldots, r_{n}\right) r_{1}^{m-i} .
$$

So (3.4) holds for $k=m$. Also, by induction, it holds for all $k \geq 0$.

Using the above structural lemma about $\hat{h}_{k}$, it is easy to derive the convergence property of $\hat{B}_{m}$.

Theorem 3.2. Let $s^{\prime}$ be the multiplicity of root $\theta_{1}$, i.e., $\theta_{1}=\theta_{2}=\cdots=\theta_{s^{\prime}}$, and $\theta_{i} \neq \theta_{1}$ for $i=s^{\prime}+1, \ldots, n$. Define $r_{j}=1 /\left(x-\theta_{j}\right), j=1, \ldots, n$.

When $s^{\prime}=s, \hat{B}_{m}(x)$ has an order of convergence $m$ for $\theta_{1}$, and

$$
\lim _{x \rightarrow \theta_{1}} \frac{\hat{B}_{m}(x)-\theta_{1}}{\left(x-\theta_{1}\right)^{m}}=\hat{h}_{m-1}\left(\left(\theta_{1}-\theta_{s^{\prime}+1}\right)^{-1}, \ldots,\left(\theta_{1}-\theta_{n}\right)^{-1}\right) .
$$

When $s^{\prime} \neq s, \hat{B}_{m}(x)$ has at most linear convergence for $\theta_{1}$, and

$$
\lim _{x \rightarrow \theta_{1}} \frac{\hat{B}_{m}(x)-\theta_{1}}{x-\theta_{1}}=\frac{s^{\prime}-s}{s^{\prime}+s(m-2)} .
$$

Proof. When $s^{\prime}=s$, equation (3.4) becomes

$$
\hat{h}_{k}\left(r_{1}, \ldots, r_{n}\right)=\sum_{i=0}^{k} \hat{h}_{i}\left(r_{s^{\prime}+1}, \ldots, r_{n}\right) r_{1}^{k-i} .
$$


From (3.3), we have

$$
\begin{aligned}
\hat{B}_{m}(x)-\theta_{1} & =x-\theta_{1}-\left(x-\theta_{1}\right) \frac{\left(x-\theta_{1}\right)^{m-2} \hat{h}_{m-2}\left(r_{1}, \ldots, r_{n}\right)}{\left(x-\theta_{1}\right)^{m-1} \hat{h}_{m-1}\left(r_{1}, \ldots, r_{n}\right)} \\
& =\left(x-\theta_{1}\right)\left(1-\frac{\sum_{i=0}^{m-2} \hat{h}_{i}\left(r_{s^{\prime}+1}, \ldots, r_{n}\right)\left(x-\theta_{1}\right)^{i}}{\sum_{i=0}^{m-1} \hat{h}_{i}\left(r_{s^{\prime}+1}, \ldots, r_{n}\right)\left(x-\theta_{1}\right)^{i}}\right) \\
& =\left(x-\theta_{1}\right)^{m} \frac{\hat{h}_{m-1}\left(r_{s^{\prime}+1}, \ldots, r_{n}\right)}{\sum_{i=0}^{m-1} \hat{h}_{i}\left(r_{s^{\prime}+1}, \ldots, r_{n}\right)\left(x-\theta_{1}\right)^{i}} .
\end{aligned}
$$

Hence,

$$
\begin{aligned}
\lim _{x \rightarrow \theta_{1}} \frac{\hat{B}_{m}(x)-\theta_{1}}{\left(x-\theta_{1}\right)^{m}} & =\lim _{x \rightarrow \theta_{1}} \frac{\hat{h}_{m-1}\left(r_{s^{\prime}+1}, \ldots, r_{n}\right)}{1+\sum_{i=1}^{m-1} \hat{h}_{i}\left(r_{s^{\prime}+1}, \ldots, r_{n}\right)\left(x-\theta_{1}\right)^{i}} \\
& =\hat{h}_{m-1}\left(\left(\theta_{1}-\theta_{s^{\prime}+1}\right)^{-1}, \ldots,\left(\theta_{1}-\theta_{n}\right)^{-1}\right) .
\end{aligned}
$$

When $s^{\prime} \neq s$, by (3.3) and (3.4) we have

$$
\begin{aligned}
\hat{B}_{m}(x)-\theta_{1} & =x-\theta_{1}-\left(x-\theta_{1}\right) \frac{\left(x-\theta_{1}\right)^{m-2} \hat{h}_{m-2}\left(r_{1}, \ldots, r_{n}\right)}{\left(x-\theta_{1}\right)^{m-1} \hat{h}_{m-1}\left(r_{1}, \ldots, r_{n}\right)} \\
& =\left(x-\theta_{1}\right)\left(1-\frac{\left(m-3+s^{\prime} / s\right)_{m-2} /(m-2) !+\sum_{i=1}^{m-2} a_{m-2, i}\left(x-\theta_{1}\right)^{i}}{\left(m-2+s^{\prime} / s\right)_{m-1} /(m-1) !+\sum_{i=1}^{m-1} a_{m-1, i}\left(x-\theta_{1}\right)^{i}}\right) .
\end{aligned}
$$

Hence,

$$
\lim _{x \rightarrow \theta_{1}} \frac{B_{m}(x)-\theta_{1}}{x-\theta_{1}}=1-\frac{\left(m-3+s^{\prime} / s\right)_{m-2} /(m-2) !}{\left(m-2+s^{\prime} / s\right)_{m-1} /(m-1) !}=\frac{s^{\prime}-s}{s^{\prime}+s(m-2)} .
$$

Remark 3.3. When $m=2$ and $s^{\prime} \leq s / 2, \hat{B}_{m}(x)$ does not converge to $\theta_{1}$ since

$$
\left|\frac{s^{\prime}-s}{s^{\prime}+s(m-2)}\right| \geq 1 \text {. }
$$

3.2. An efficient algorithm for computing $\hat{B}_{m}$. Let $\hat{Q}(t)$ and $\hat{H}(t)$ be the generating functions for $\hat{q}_{r}$ and $\hat{h}_{r}$, respectively. That is,

$$
\hat{Q}(t)=\sum_{r \geq 1} \hat{q}_{r} t^{r-1} \quad \text { and } \quad \hat{H}(t)=\sum_{r \geq 0} \hat{h}_{r} t^{r} .
$$

By (3.1), we have

$$
\hat{Q}(t)=\frac{1}{s} Q(t)=\frac{E^{\prime}(-t)}{s E(-t)},
$$

and recursion (3.2) implies

$$
\hat{Q}(t)=\frac{\hat{H}^{\prime}(t)}{\hat{H}(t)} .
$$

Thus,

$$
s E(-t) \hat{H}^{\prime}(t)=E^{\prime}(-t) \hat{H}(t),
$$

which gives rise to the following recursion:

$$
\hat{h}_{i}=\frac{1}{s \cdot i} \sum_{r=0}^{i-1}(-1)^{i-r-1}(i-r+s \cdot r) e_{i-r} \hat{h}_{r} \quad \text { for } \quad i=1,2, \ldots .
$$


Given an input $x_{0}$, an efficient algorithm to compute $\hat{B}_{m}\left(x_{0}\right)$ is as follows:

Algorithm 2: Evaluation of the $m$-th order iteration function $\hat{B}_{m}$ for roots of multiplicity $s$ at $x_{0}$.

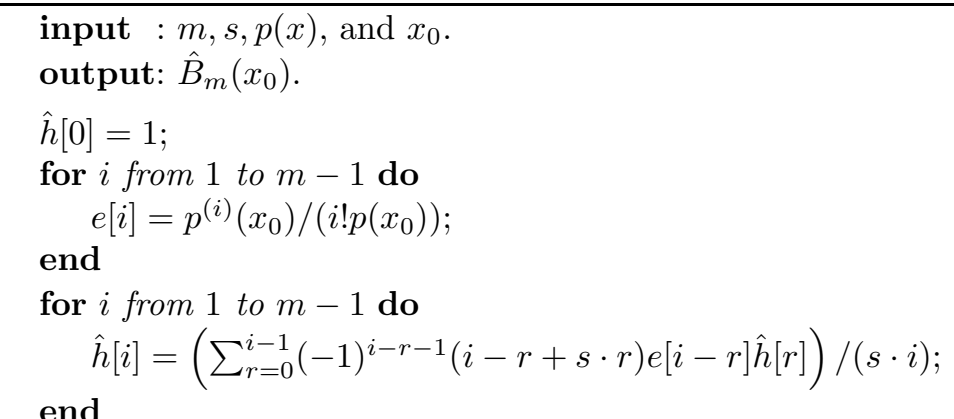

end

output $\hat{B}_{m}\left(x_{0}\right)=x_{0}-\hat{h}[m-2] / \hat{h}[m-1] ;$

Remark 3.4. For a polynomial $p(x)$ of degree $n$, the normalized derivatives $p^{(i)}\left(x_{0}\right) / i$ ! can be evaluated in $O\left(n \log ^{2} n\right)$ time (see Kung [12]). Furthermore, $\hat{h}[m]$, the $m$-th term of a sequence defined via a homogeneous linear recurrence relation, can be computed in $O(n \log n \log m)$ arithmetic operations (see Fiduccia [2]). Therefore, the computational complexity of $\hat{B}_{m}\left(x_{0}\right)$ is $O(n \log n(\log m+\log n))$.

Using the above algorithm, we can also derive the closed form formulas for $\hat{B}_{2}, \hat{B}_{3}$ and $\hat{B}_{4}$ :

$$
\begin{aligned}
& \hat{B}_{2}(x)=x-s \frac{p(x)}{p^{\prime}(x)} \\
& \hat{B}_{3}(x)=x-2 s p(x) \frac{p^{\prime}(x)}{(1+s) p^{\prime}(x)^{2}-s p(x) p^{\prime \prime}(x)} \\
& \hat{B}_{4}(x)=x-3 s p(x) \frac{(1+s) p^{\prime}(x)^{2}-s p(x) p^{\prime \prime}(x)}{\left(2 s^{2}+3 s+1\right) p^{\prime}(x)^{3}-3 s(1+s) p(x) p^{\prime}(x) p^{\prime \prime}(x)+s^{2} p(x)^{2} p^{\prime \prime \prime}(x)} .
\end{aligned}
$$

Remark 3.5. The generalized complete symmetric functions $\hat{h}_{i}$ also admit an upper Hessenberg determinantal form

$$
\hat{h}_{i}=\operatorname{det}\left[\begin{array}{ccccc}
c(i, 1) e_{1} & c(i, 2) e_{2} & \ldots & c(i, i-1) e_{i-1} & c(i, i) e_{i} \\
1 & c(i-1,1) e_{1} & \ddots & \ddots & c(i-1, i-1) e_{i-1} \\
0 & 1 & \ddots & \ddots & \vdots \\
\vdots & \vdots & \ddots & \ddots & c(2,2) e_{2} \\
0 & 0 & \ldots & 1 & c(1,1) e_{1}
\end{array}\right] \text {, }
$$

where coefficients $c(i, j)=(j+s(i-j)) /(s \cdot i)$. This follows from the fact that by expanding the determinant along the first row we arrive at recurrence relation (3.6).

When $s=1$, all the coefficients $c(i, j)=1$ and the above formula is equivalent to the determinantal representation of Basic Family given by [7]. 
4. The connection Between $B_{m}$ And $\hat{B}_{m}$

In this section, we show that $\hat{B}_{m}$ is actually the application of $B_{m}$ to $p(x)^{1 / s}$.

We start with a generalization of Proposition 2.1.

Proposition 4.1. Let $p(x)$ be a polynomial of degree $n$ with complex coefficients, and let $\theta_{1}, \ldots, \theta_{n}$ be its complex roots. Let $f(x)=p(x)^{1 / s}$.

Define $r_{j}=1 /\left(x-\theta_{j}\right), j=1, \ldots, n$. Define

$$
\tilde{e}_{i}=\sum_{|\lambda|=i} m_{\lambda} \prod_{j=1}^{l(\lambda)} \frac{(1 / s)_{\lambda_{j}}}{\lambda_{j} !}
$$

where $m_{\lambda}$ is the monomial symmetric function corresponding to partition $\lambda=$ $\left(\lambda_{1}, \lambda_{2}, \ldots\right)$.

Then

$$
f^{(i)}(x)=i ! f(x) \tilde{e}_{i}\left(r_{1}, \ldots, r_{n}\right)
$$

where $f^{(i)}(x)$ is the $i$-th derivative of $f(x)$.

Proof. Write

$$
f(x)=c^{1 / s} \prod_{i=1}^{n}\left(x-\theta_{i}\right)^{1 / s},
$$

and apply the generalized product rule of differentiation to it.

The generating function for $\tilde{e}_{i}$ is

$$
\tilde{E}(t)=\sum_{r \geq 0} \tilde{e}_{r} t^{r}=\prod_{i \geq 1}\left(1+x_{i} t\right)^{1 / s}=E(t)^{1 / s},
$$

which is easy to verify using the power series expansion

$$
\left(1+x_{i} t\right)^{1 / s}=1+\sum_{k=1}^{\infty} \frac{(1 / s)_{k}}{k !} x_{i}^{k} t^{k} .
$$

Let $\tilde{B}_{m}$ be the iteration function obtained by applying $B_{m}$ to $p(x)^{1 / s}$. Then by Algorithm [ we have

$$
\tilde{B}_{m}(x)=x-\frac{\tilde{h}_{m-2}\left(r_{1}, \ldots, r_{n}\right)}{\tilde{h}_{m-1}\left(r_{1}, \ldots, r_{n}\right)} \quad \text { for } \quad m=2,3, \ldots,
$$

where $\tilde{h}_{0}=1$, and

$$
\tilde{h}_{i}=\sum_{r=0}^{i-1}(-1)^{i-r-1} \tilde{e}_{i-r} \tilde{h}_{r} \quad \text { for } \quad i=1,2, \ldots .
$$

Let $\tilde{H}(t)$ be the generating function for $\tilde{h}_{r}$. Then from the above recurrence relation between $\tilde{h}_{r}$ and $\tilde{e}_{r}$ we have $\tilde{H}(t)=\tilde{E}(-t)^{-1}$. Thus,

$$
\frac{\tilde{H}^{\prime}(t)}{\tilde{H}(t)}=\frac{\left[E(-t)^{-1 / s}\right]^{\prime}}{E(-t)^{-1 / s}}=\frac{E^{\prime}(-t)}{s E(-t)}=\hat{Q}(t)=\frac{\hat{H}^{\prime}(t)}{\hat{H}(t)},
$$

which implies $\tilde{H}(t)=\hat{H}(t)$, and $\tilde{h}_{r}=\hat{h}_{r}$ for $r=0,1,2, \ldots$

Therefore, $\hat{B}_{m} \equiv \tilde{B}_{m}$; i.e., $\hat{B}_{m}$ is the application of $B_{m}$ to $p(x)^{1 / s}$. 
Remark 4.2. $\tilde{e}_{r}, r=0,1,2, \ldots$ are generalized elementary symmetric functions, and the recurrence relations among $\tilde{e}_{r}, \hat{h}_{r}$ and $\hat{q}_{r}$ are the same as those among the standard $e_{r}, h_{r}$ and $q_{r}$.

An explicit formula for $\hat{B}_{m}$ can be obtained by expanding $\hat{h}_{m-2}$ and $\hat{h}_{m-1}$ into functions of $p(x)$ and its derivatives.

Using the relation between generating functions $\hat{H}(t)$ and $E(t)$,

$$
\hat{H}(t)=\sum_{r \geq 0} \hat{h}_{r} t^{r}=E(-t)^{-1 / s}
$$

we can express $\hat{h}_{r}$ as a polynomial in $e_{i}$ 's directly.

Define

$$
T=\sum_{r=1}^{\infty} e_{r}(-t)^{r}
$$

then $E(-t)=1+T$, and by Taylor expansion we have

$$
\hat{H}(t)=(1+T)^{-1 / s}=1+\sum_{i=1}^{\infty} \frac{(-1 / s)_{i}}{i !} T^{i}
$$

Thus,

$$
\begin{aligned}
& \hat{h}_{k}=(-1)^{k} \sum_{\substack{|\lambda|=k \\
\lambda=\left(\lambda_{1}^{a_{1}} \ldots \lambda_{v}^{a_{v}}\right)}} \frac{(-1 / s)_{l(\lambda)}}{l(\lambda) !} \frac{l(\lambda) !}{\prod_{j=1}^{v} a_{j} !} e_{\lambda} \\
& =(-1)^{k} \sum_{\substack{|\lambda|=k \\
\lambda=\left(\lambda_{1}^{a_{1}} \ldots \lambda_{v}^{a_{v}}\right)}} \frac{(-1 / s)_{l(\lambda)}}{\prod_{j=1}^{v} a_{j} !} e_{\lambda} . \\
& =(-1)^{k} \sum_{\substack{|\lambda|=k \\
\lambda=\left(\lambda_{1}^{a_{1}} \ldots \lambda_{v}^{a_{v}}\right)}}(-1 / s)_{l(\lambda)} \prod_{j=1}^{v} \frac{\left[p^{\left(\lambda_{j}\right)}(x)\right]^{a_{j}}}{a_{j} !\left[\lambda_{j} ! p(x)\right]^{a_{j}}} .
\end{aligned}
$$

[1] X. Buff and C. Henriksen, On König's root-finding algorithms, Nonlinearity, 16 (2003) 9891015. MR:1975793(2004c:37086)

[2] C.M. Fiduccia, An efficient formula for linear recurrences, SIAM J. Comput., 14 (1985) 106112. MR774930 (86h:39001)

[3] E. Hansen and M. Patrick, A family of root finding methods, Numer. Math., 27 (1976/77) 257-269. MR0433858 (55:6829)

[4] Y. Jin and B. Kalantari, Symmetric functions and root-finding algorithms, Adv. Appl. Math., 34 (2005) 156-174. MR2102280 (2006a:05167)

[5] Y. Jin, Combinatorics of polynomial root-finding algorithms, Ph.D. dissertation, Dept. of Computer Science, Rutgers University, New Brunswick, NJ, October 2005.

[6] Y. Jin, On efficient computation and asymptotic sharpness of Kalantari's bounds for zeros of polynomials, Math. Comp., 75 (2006) 1905-1912. MR.2240641 (2007e:65046)

[7] B. Kalantari, I. Kalantari, and R. Zaare-Nahandi, A basic family of iteration functions for polynomial root finding and its characterizations, J. Comput. Appl. Math., 80 (1997) 209-226. MR:1455244 (98d:65066)

[8] B. Kalantari, Generalization of Taylor's theorem and Newton's method via a new family of determinantal interpolation formulas and its applications, J. Comput. Appl. Math., 126 (2000) 287-318. MR 1806762 (2001m:41035)

[9] B. Kalantari and Y. Jin, On extraneous fixed-points of the basic family of iteration functions, BIT, 43 (2003) 453-458. MR2010487 (2004g:65066) 
[10] B. Kalantari, On homogeneous linear recurrence relations and approximation of zeros of complex polynomials, Unusual applications of number theory, 125-143, DIMACS Ser. Discrete Math. Theoret. Comput. Sci., 64, Amer. Math. Soc., Providence, RI, 2004. MR2063208 (2005d:12001)

[11] B. Kalantari, An infinite family of bounds on zeros of analytic functions and relationship to Smale's bound, Math. Comp., 74 (2005) 841-852. MR2114651 (2005k:65093)

[12] H.T. Kung, A new upper bound on the complexity of derivative evaluation, Inform. Process. Lett., 2 (1973) 146-147. MR0347135 (49:11855)

[13] I.G. MacDonald, Symmetric Functions and Hall Polynomials, 2nd Edition, The Clarendon Press, Oxford University Press, Oxford, 1995. MR.1354144 (96h:05207)

[14] E. Schröder, Ueber unendlich viele Algorithmen zur Auflösung der Gleichungen, Math. Ann., 2 (1870) 317-365. (English translation, "On infinitely many algorithms for solving equations", by G.W. Stewart, TR-92-121, Institute for Advanced Computer Studies, University of Maryland, College Park, MD, 1992.) MR.1509664

Quantitative Research, Interest Rate Derivatives, J.P. Morgan, 270 Park Avenue, New York, New York 10017

E-mail address: yi.x.jin@jpmorgan.com

Department of Computer Science, Rutgers University (New Brunswick), Hill CenterBusch Campus, 110 Frelinghuysen Road, Piscataway, New Jersey 08854

E-mail address: kalantar@cs.rutgers.edu 